\title{
A comparison of BMP2 delivery by coacervate and gene therapy for promoting human muscle-derived stem cell-mediated articular cartilage repair
}

\author{
Xueqin Gao ${ }^{1,2,3}$, Haizi Cheng ${ }^{1}$, Hassan Awada ${ }^{4}$, Ying Tang ${ }^{5}$, Sarah Amra ${ }^{1}$, Aiping Lu ${ }^{1,2,3}$, Xuying Sun ${ }^{1}$, Guijin Lv' \\ Charles Huard', Bing Wang ${ }^{5}$, Xiaohong $\mathrm{Bi}^{6}$, Yadong Wang ${ }^{4}$ and Johnny Huard ${ }^{1,2,3^{*}}$ (D)
}

\begin{abstract}
Background: Osteoarthritis and cartilage injury treatment is an unmet clinical need. Therefore, development of new approaches to treat these diseases is critically needed. Previous work in our laboratory has shown that murine muscle-derived stem cells (MDSCs) can efficiently repair articular cartilage in an osteochondral and osteoarthritis model. However, the cartilage repair capacity of human muscle-derived stem cells has not been studied which prompt this study.
\end{abstract}

Method: In this study, we tested the in vitro chondrogenesis ability of six populations of human muscle-derived stem cells (hMDSCs), before and after lenti-BMP2/GFP transduction using pellet culture and evaluated chondrogenic differentiation of via histology and Raman spectroscopy. We further compared the in vivo articular cartilage repair of hMDSCs stimulated with BMP2 delivered through coacervate sustain release technology and lenti-viral gene therapy-mediated gene delivery in a monoiodoacetate (MIA)-induced osteoarthritis (OA) model. We used microCT and histology to evaluate the cartilage repair.

Results: We observed that all hMDSCs were able to undergo chondrogenic differentiation in vitro. As expected, lenti-BMP2/GFP transduction further enhanced the chondrogenic differentiation capacities of hMDSCs, as confirmed by Alcian blue and Col2A1staining as well as Raman spectroscopy analysis. We observed through micro-CT scanning, Col2A1 staining, and histological analyses that delivery of BMP2 with coacervate could achieve a similar articular cartilage repair to that mediated by hMDSC-LBMP2/GFP. We also found that the addition of soluble fms-like tyrosine kinase-1 (SFLT-1) protein further improved the regenerative potential of hMDSCS/BMP2 delivered through the coacervate sustain release technology. Donor cells did not primarily contribute to the repaired articular cartilage since most of the repair cells are host derived as indicated by GFP staining.

Conclusions: We conclude that the delivery of hMDSCs and BMP2 with the coacervate technology can achieve a similar cartilage repair relative to lenti-BMP2/GFP-mediated gene therapy. The use of coacervate technology to deliver BMP2/ sFLT1 with hMDSCs for cartilage repair holds promise for possible clinical translation into an effective treatment modality for osteoarthritis and traumatic cartilage injury.

Keywords: Bone morphogenetic proteins 2, Human muscle-derived stem cells, Coacervate, Osteoarthritis, Soluble fms-like tyrosine kinase-1, Cartilage repair

\footnotetext{
*Correspondence: jhuard@sprivail.org

'Department of Orthopaedic Surgery, University of Texas Health Science

Center at Houston, Houston, TX, USA

${ }^{2}$ Institute of Molecular Medicine, McGovern Medical School, University of

Texas Health Science Center at Houston, Houston, TX, USA

Full list of author information is available at the end of the article
}

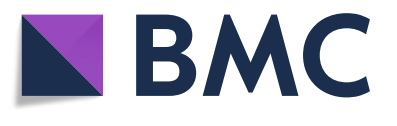

(c) The Author(s). 2019 Open Access This article is distributed under the terms of the Creative Commons Attribution 4.0 International License (http://creativecommons.org/licenses/by/4.0/), which permits unrestricted use, distribution, and reproduction in any medium, provided you give appropriate credit to the original author(s) and the source, provide a link to the Creative Commons license, and indicate if changes were made. The Creative Commons Public Domain Dedication waiver (http://creativecommons.org/publicdomain/zero/1.0/) applies to the data made available in this article, unless otherwise stated. 


\section{Background}

Osteoarthritis is a degenerative joint disease caused by loss of articular cartilage which affects $1 / 3$ of the population aged 65 and older. It severely affects the daily activities of the patient. In the process of seeking a cure for this degenerative disease, clinicians and researcher have explored many different approaches for treatment of osteoarthritis. Using stem cell and growth factor gene therapy to regenerate articular cartilage has been one important field to develop approaches for treatment of osteoarthritis. It has been shown that bone marrow mesenchymal stem cell (BMMSCs) can repair articular cartilage when combined with certain scaffold materials and growth factors, such as bone morphogenetic protein 2 and 4 (BMP2,BMP4), Indian Hedgehog, and the transcription factor SOX-9 $[1,2]$. The beneficial effects of BMMSCs have been shown to be not only related to their ability for direct differentiation to the chondrogenic lineage, but also to their immunosuppressive effects [3]. However, beneficial effects have not always been achieved, as was shown in a large animal (horse) model for which adding BMMSCs did not demonstrate better cartilage repair [4]. Adipose-derived stem cells have also been studied for cartilage repair when delivered with growth factors for gene therapy using different vectors or scaffolds [5-9]. A systematic review by Goldberg et al. demonstrated the advances of stem cells and cartilage repair [10]. Despite progress made towards the repair of cartilage, clinical application of these new treatments is still very limited. Muscle-derived stem cells (MDSCs) represent one kind of stem cell that has great potential for tissue engineering for musculoskeletal regeneration. Genetically modified murine MDSCs have been shown to effectively repair articular cartilage both in a monoiodoacetate (MIA)-induced osteoarthritis model and osteochondral defect model [11]. Also, blocking angiogenesis with soluble fms-like tyrosine kinase-1 (sFLT1)-transduced murine MDSCs has been shown to further enhance the cartilage repair capacity of bone morphogenetic protein 4 (BMP4)-transduced MDSCs $[12,13]$. However, mMDSCs required gene modification to effectively undergo chondrogenic differentiation and promote articular cartilage repair. Human musclederived stem cells (hMDSCs) have been shown to be able to differentiate into chondrogenic, osteogenic, adipogenic, and myogenic lineages in vitro and have been able to enhance bone regeneration in vivo, when transduced with lentivirus expressing BMP2 [14]. It has also been shown that BMP2-transduced hMDSCs can repair critical size bone defects as efficiently as human BMMSCs [15]. HMDSCs' bone regeneration capacities do not decline with aging [16]. However, whether hMDSCs could repair articular cartilage in vivo is still unknown. Coacervate is a slow release material formed by mixing solutions of heparin and a polycation, polyethylene argininylaspartate diglyceride (PEAD). It has been used for the delivery of fibroblast growth factor 2 (FGF2) to enhance angiogenesis in vivo successfully [17]. Coacervation is a self-assembly process driven by electrostatic forces that in this case allows near quantitative capturing of heparin-binding growth factors into the coacervate phase. Subsequently, it has been used to deliver different growth factors for cardiac infarction repair and effective wound healing [18-24]. It also has been utilized to sustained release of BMP2 for promoting murine MDSC-mediated ectopic bone formation [25]. In the current study, we compared gene therapy and a coacervate sustained delivery system to deliver BMP2 to test whether using coacervate-delivered BMP2 could enhance hMDSC-mediated cartilage repair using an MIAinduced osteoarthritis model, and the results were compared to lenti-BMP2/GFP-transduced hMDSC-mediated gene therapy.

\section{Materials and methods}

The use of human tissue was approved by the IRB at the University of Texas Health Science Center at Houston (UTHealth), and all animal experiments and procedures were approved by the IACUC at UTHealth (AWC-15-0072).

\section{Cell isolation}

Six populations of hMDSCs were isolated via a modified preplate technique, as previously described [26], from skeletal muscle biopsies purchased from the National Disease Research Interchange (NDRI). This pre-plate technique we used for isolation of MDSCs was modified from previously reported protocol [27-29]. Briefly, the muscle tissues purchased from NDRI were rinsed with Hanks Balanced Salt Solution (HBSS), and the fat and connective tissues were removed. Muscle tissues were cut into small pieces with scissors into slurry $(1 \mathrm{~mm}$ in size) in HBSS solution. Then, the tissue slurry was centrifuged to remove HBSS, and pellets were weighed, and $1 \mathrm{ml}$ of Collagenase XI was used for $0.1 \mathrm{~g}$ muscle. The tissues were digested with collagenase XI (0.2\% weight/ volume) (Sigma-Aldrich, Cat. \#C7657), dispase (2.4 U/ ml) (Invitrogen, Cat. \#17105-041), and Trypsin-EDTA (0.1\% weight/volume, Invitrogen, Cat. \#15400-054) at $37^{\circ} \mathrm{C}$. The digestion time was $1 \mathrm{~h}$ for collagenase XI, 45 min for dispase, and $30 \mathrm{~min}$ for Trypsin-EDTA. The enzyme was removed by centrifugation at $2630 \mathrm{~g}$ for $5 \mathrm{~min}$ for each step. We added the dispase digestion step in our protocol compared to previous protocol. The cells were seeded in collagen I coated flasks (Sigma-Aldrich, Cat. \#C9791) and incubated at $5 \% \mathrm{CO}_{2}$ and $37^{\circ} \mathrm{C}$ for 2 $\mathrm{h}$. The un-adhered cells were transferred to another new collagen-coated flask. This step was repeated 5 more times until we obtained the preplate 6 (pp6). The pp6 
cells eventually adhered and grew and were called hMDSCs [26]. The 6 populations of hMDSCs included three young populations (31-year-old female, hMDSC1; 21-year-old male, hMDSC2; 23-year-old male, hMDSC3) and three old populations (76-year-old female, hMDSC4; 78-year-old male, hMDSC5; 80-year-old male, hMDSC6) respectively. The hMDSCs were grown and maintained in proliferation medium (PM) that contained high glucose DMEM (Invitrogen) supplemented with 20\% fetal bovine serum (FBS), 1\% chicken embryo extract (CEE), and $1 \%$ penicillin/streptomycin.

\section{Construction of the lenti-viral-BMP2/GFP vector}

A lenti-viral vector encoding for human BMP2 gene under the control of the human cytomegalovirus (CMV) promoter with a green fluorescent protein (GFP) tag, which was separated by an internal ribosome entry site (IRES) from the target gene, was constructed in collaboration with Dr. Bing Wang's Laboratory. The GFP tag allowed us to monitor transduction efficiency and use fluorescenceactivated cell sorting (FACS) to select transduced cells. Lenti-GFP (LGFP) and lenti-BMP2/GFP (LBMP2/GFP) viral vectors were packaged using $293 \mathrm{~T}$ cells (ATCC) using established protocol.

\section{Cell transduction}

Human MDSCs were transduced with LBMP2/GFP or LGFP virus in the presence of polybrene $(8 \mu \mathrm{g} / \mathrm{ml})$ using 1:4 and 1:12 dilution with proliferation medium respectively at passage $8-10$ for $16 \mathrm{~h}$. At $24 \mathrm{~h}$ after transduction, transduction efficiency was observed under a fluorescent microscope. The transduction efficiency was about 50$60 \%$. Cells were passaged 2 times after transduction and then subjected to GFP cell sorting (FACS) based on GFP fluorescence. After cell sorting, the cells were expanded in proliferation media. Supernatants were collected from different passages of each population and BMP2 secretion levels were measured using an ELISA (DBP200, R\&D system). One population of young female cells (hMDSC1) was transduced with both LGFP and LBMP2/GFP for in vivo studies.

\section{In vitro chondrogenesis}

In vitro chondrogenesis assay was performed for nontransduced or LBMP2/GFP-transduced hMDSCs using a 3D pellet culture method, as previously described [30]. Briefly, 6 populations of non-transduced cells and 6 populations of LBMP2/GFP-transduced cells were cultured in proliferation medium and expanded. $1.25 \times 10^{5}$ cells from each population were aliquoted to $15 \mathrm{ml}$ tubes in 4 replicates/population. The cells were centrifuged at $800 g$ for $5 \mathrm{~min}$ and resuspended with complete chondrogenic medium (StemPro ${ }^{\circ}$ Chondrogenesis Differentiation Kit, A1007101, ThermoFisher Scientific). The cells were then centrifuged at $500 \mathrm{~g}$ for $5 \mathrm{~min}$, and the lids of tubes were loosened $1 / 4$ turn to allow for oxygen exchange. Using this method, cells usually form pellets around 3 days of culture. Chondrogenic medium was changed every 2-3 days for 24 days. The pellets were fixed with neutral buffered formalin (NBF), rinsed once with PBS, then embedded in NEG freezing medium, snap frozen in liquid nitrogen, and stored at $-80^{\circ} \mathrm{C}$ until sectioning, at which time $8-\mu \mathrm{m}$ cryosections were cut. Pellets' cultures were repeated three times for each population. Alcian blue staining was performed using online protocol (http:// www.ihcworld.com/_protocols/special_stains/alcian_ blue.htm.). Images were captured using a NIKON Cil microscope, and blue matrix was quantified using the NIKON NIS Element software. Collagen type II alpha 1 (Col2A1) immunohistochemistry (IHC) was also performed using goat anti-Col2A1 (SC7764, 1:50, Santa Cruz Biotechnology). In addition, Raman spectroscopy was utilized to quantitate sulfated cartilage matrix (proteoglycan aggrecan) at the Raman band $\sim 1060 \mathrm{~cm}^{-1}$ (sulfate) and collagen at Raman band $\sim 856 \mathrm{~cm}^{-1}$ (Proline) for the chondrogenic pellets derived from hMDSC5 and hMDSC6 before fixation by Dr. Xiaohong Bi's laboratory.

\section{Preparation of coacervate and binding of BMP2 and sFLT1}

Preparation of coacervate and binding of BMP2 and sFLT1 was carried out as follows. BMP2 (120-02C, PeproTech) and sFLT1 (ab54346, Abcam) were purchased and resuspended according to the manufacturer's protocol to a concentration of $100 \mathrm{ng} / \mu \mathrm{l}$ in phosphatebuffered saline (PBS). Coacervate was formed using heparin and PEAD, which was synthesized by Dr. Yadong Wang's lab, as previously described [31, 32]. We engineered our controlled delivery system to be more stable than the typical coacervate after a selection process that paired the polycation, PEAD, with heparin. We have previously demonstrated that the coacervate was present after 4 weeks in an infarcted myocardium [33]. BMP2 (500 ng) alone, or BMP2 plus sFLT1 (500 ng each), was first mixed with $12.5 \mu \mathrm{l}$ of heparin $(2 \mathrm{mg} / \mathrm{ml})$ to allow proteins to bind to heparin, followed by addition of $12.5 \mu \mathrm{l}$ of PEAD $(10 \mathrm{mg} / \mathrm{ml})$, and the complex became turbid which indicated the formation of coacervate. Then, each coacervate mixture was ready for combining with the different cell populations, as described below.

\section{In vivo articular cartilage (AC) repair using MIA-induced osteoarthritis model}

In vivo cartilage repair was investigated using an MIAinduced global osteoarthritis model with delivery of BMP2 and hMDSCs using coacervate, and this delivery method was compared with LBMP2/GFP gene therapy. 
Thirty male nude rats (Taconic) of 12 weeks old were injected with $0.3 \mathrm{mg} / 150 \mathrm{~g}$ body weight MIA in $50 \mu \mathrm{l}$ volume in the right knee (injured) according to our published paper [12], and the left knee (uninjured) was used as the normal control per our animal protocol approved by the IACUC at UTHealth. Two weeks after MIA injection, the rats were divided into 5 groups and injected in the injured knee joint with different combinations of cells/proteins or complexes (as stated below). Nude rats were divided into the following 5 groups ( $N=6$ each group).

PBS + coacervate group: PEAD $(12.5 \mu \mathrm{l})$ was added to heparin $(12.5 \mu \mathrm{l})$, and then PBS $(25 \mu \mathrm{l})$ was added, followed by mixing and injection.

$B M P 2+P B S+h M D S C-L G F P\left(1 \times 10^{6}\right)$ group: BMP2 (500 ng in $5 \mu \mathrm{l})$ was added to PBS $(25 \mu \mathrm{l})$ and then added to the hMDSC-LGFP cell suspension $\left(1 \times 10^{6}\right.$ cells in $20 \mu \mathrm{l}$ PBS), followed by mixing prior to injection.

$P B S+h M D S C-L B M P 2 / G F P\left(1 \times 10^{6}\right)$ group: PBS $(25 \mu \mathrm{l})$ was added to $25 \mu \mathrm{l}$ of cell suspension, for a total of $50 \mu \mathrm{l}$, and mixed prior to injection.

BMP2 + coacervate + hMDSC-LGFP $\left(1 \times 10^{6}\right)$ group: BMP2 (500 ng in $5 \mu \mathrm{l})$ was mixed with heparin $(12.5 \mu \mathrm{l})$, then PEAD was added $(12.5 \mu \mathrm{l})$, and the complex was mixed with hMDSCs $\left(1 \times 10^{6}\right)$ in $20 \mu \mathrm{l}$ PBS before injection.

$B M P 2+$ sFLT1 + coacervate + hMDSC-LGFP group: BMP2 (500 ng in $5 \mu \mathrm{l})$ and sFLT1 $(500 \mathrm{ng}$ in $5 \mu \mathrm{l})$ were mixed with heparin $(12.5 \mu \mathrm{l})$ and then PEAD $(12.5 \mu \mathrm{l})$ was added, and then the protein-loaded complex was mixed with the hMDSC-LGFP cell suspension $\left(1 \times 10^{6}\right.$ in $15 \mu \mathrm{l}$ PBS) just prior to knee joint injection. The nude rats were sacrificed at 12 weeks post-knee joint cell injection, and both the injured and uninjured knees were harvested and then fixed in NBF for 3 days for subsequent microCT and histology.

\section{MicroCT scanning and analysis}

After fixation, both injured and uninjured knees were scanned using microCT (Viva CT 40, Scanco Medical) without contrast using $70 \mathrm{kvp}, 112 \mu \mathrm{A}$, and a $30-\mu \mathrm{m}$ voxel size. 3D images of the whole knee joint were reconstructed using Gauss $=0.8$, Sigma $=1$, and a threshold of 200 using the same dimensions for subsequent analysis. After 3D reconstruction, the empty gap of each knee joint of 3D image was measured using image J software. The bigger gap indicated more severe the cartilage erosion. The knee joint gap differences were calculated using injured knee gap subtracted non-injured knee of the same rat and compared between groups.

\section{Histology}

After microCT, the knee joints were decalcified using 10\% ethylenediaminetetraacetic acid disodium (EDTA) plus 1\% sodium hydroxide for 3 months. Whole knee joints were cut in the middle sagittally, dehydrated, and then paraffin embedded so that the middle of the joint (groove level) and the edge of the joint (condyle level) could be viewed. Both levels of sections were used for quantification and histology score. Paraffin sections of $5 \mu \mathrm{m}$ were cut. H\&E staining and Alcian blue staining were performed per the following IHC WORLD protocols respectively: http://www.ihcworld.com/_protocols/special_stains/h\&e_ellis.htm

http://www.ihcworld.com/_protocols/special_stains/ alcian_blue.htm.

Toluidine blue staining was performed using IHC world protocol:

http://www.ihcworld.com/_protocols/special_stains/ toluidine_blue.htm

Images were captured for entire area of cartilage surface of each animal at both middle (groove) and edge (condyle) at $\times 200$ magnification. The summed area of each pathological change was measured with Image J for scoring. Histology score was given using Osteoarthritis Research Society International (OARSI) grading (1-6) and staging (1-4) criteria [34]. The grading and staging were performed blindly. If the score is higher, it indicates worse cartilage repair.

\section{Immunohistochemistry}

IHC staining of GFP-positive cells was used to reveal donor cells in the regenerated cartilage and Col2 staining was used to detect specific cartilage matrix collagen 2. Briefly, after deparaffinization, washing, and blocking with $5 \%$ donkey serum in PBS, sections were incubated with rabbit anti-GFP antibody (ab290, Abcam, 1:1000 dilution) and rabbit anti-Col2 (ab34712, Abcam: 1:400 dilution) in 5\% donkey serum overnight. For Col2 staining, antigen retrieval was performed using $2 \%$ hyaluronidase (H3506-5G, Sigma,) in PBS at room temperature for 30 min, followed by washing with PBS three times before blocking and incubation with primary antibody. The following day, sections were treated with $0.5 \% \mathrm{H}_{2} \mathrm{O}_{2}$ in PBS for $30 \mathrm{~min}$ at room temperature, washed in $\mathrm{PBS}$, and then incubated with goat anti-rabbit biotin (BA 1000, Vector Laboratories, Burlingame, CA, USA, 1:200 dilution) for $2 \mathrm{~h}$ at room temperature. After three washes, each slide was incubated with $A B C$ reagent (PK 7200, Elite $A B C$ kits, Vector Laboratories) for $2 \mathrm{~h}$ at room temperature. After three washes with PBS, diaminobenzidine (DAB) staining (SK-4100, Vector Laboratories) was used to visualize the GFP-positive cells. Hematoxylin (H-3404, Vector laboratories) counterstaining was performed following the DAB color reaction.

\section{Statistical analysis}

One-way analysis of variance (ANOVA) followed by Tukey's post hoc multiple test was used to analyze 
multiple quantitative data using GraphPad Prism 7. Two-side Student $T$ test was used to compare the two groups. Wilcoxon rank-sum non-parametric test was used for microCT and histology score analysis due to high deviation of the parameters. In brief, the two comparison group values were ranked from small to large, and the numerical rank sum of each group was compared to the Wilcoxon rank-sum table; the $P$ value was determined based on the upper tail and lower tails of the comparison groups. If the sum of one group was larger than the upper tail value, then we deemed $P<0.05$ or $P<0.01$ to be statistically different. Similarly, if the sum of one group was lower than lower tail probability value, we also deemed $P<0.05$ or $P<0.01$ was statistically different. Overall, a value of $P<0.05$ was considered statistically significant for all statistical analysis method.

\section{Results}

Lenti-viral transduction efficiency of hMDSCs

We found that lenti-BMP2/GFP transduction efficiency was about $50-60 \%$ for 6 populations of hMDSCs. The cells proliferated well at 1:4 dilution. After GFP flow cytometry cell sorting, the GFP-positive rate was increased to $90-95 \%$ after transduction; however, GFP fluorescent intensity became weaker after sorting (Fig. 1a). For the
lenti-GFP transduction, the transduction efficiency was about $80 \%$ when we used 1:12 dilution. After GFP sorting, the GFP-positive rate increased to around 95\% based on fluorescent imaging (Fig. 1b). The BMP2 secretion levels for the sorted lenti-BMP2/GFP-transduced cells range from 1171 to $5123 \mathrm{pg} / \mathrm{million}$ cells $/ 24 \mathrm{~h}$. The level of BMP2 secretion by the transduced was the highest with the hMDSC population 1 (hMDSC1) and the lowest with hMDSC population 5 (hMDSC5) (Fig. 1c).

\section{LBMP2/GFP-transduced hMDSCs exhibited higher} chondrogenic differentiation capacity compared to nontransduced hMDSCs

We performed 3D pellet culture assays to test the chondrogenic potential of 6 populations of human cells before and after lenti-BMP2/GFP transduction. After 24 days of culturing in chondrogenic medium, Alcian blue staining showed more Alcian blue-positive chondrogenic matrix in the LBMP2/GFP-transduced hMDSC pellets compared to non-transduced hMDSC pellets in all cell populations tested (Fig. 2a). Quantification of the percentage of blue matrix demonstrated significantly higher chondrogenesis in the LBMP2/GFP-transduced relative to non-transduced cells for all 6 populations of hMDSCs (Fig. 2b). Raman spectroscopy measurement indicated the LBMP2/GFP-

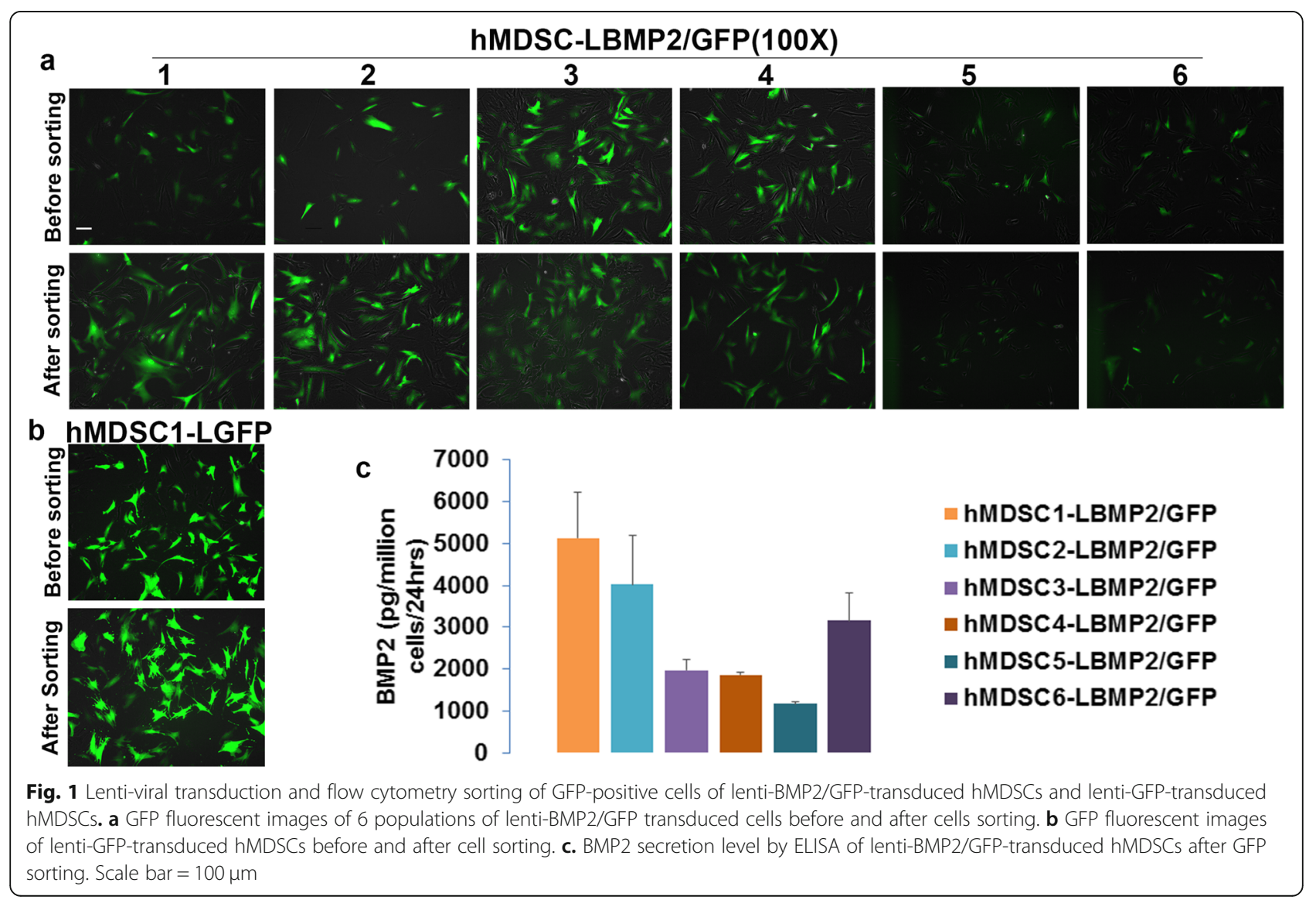




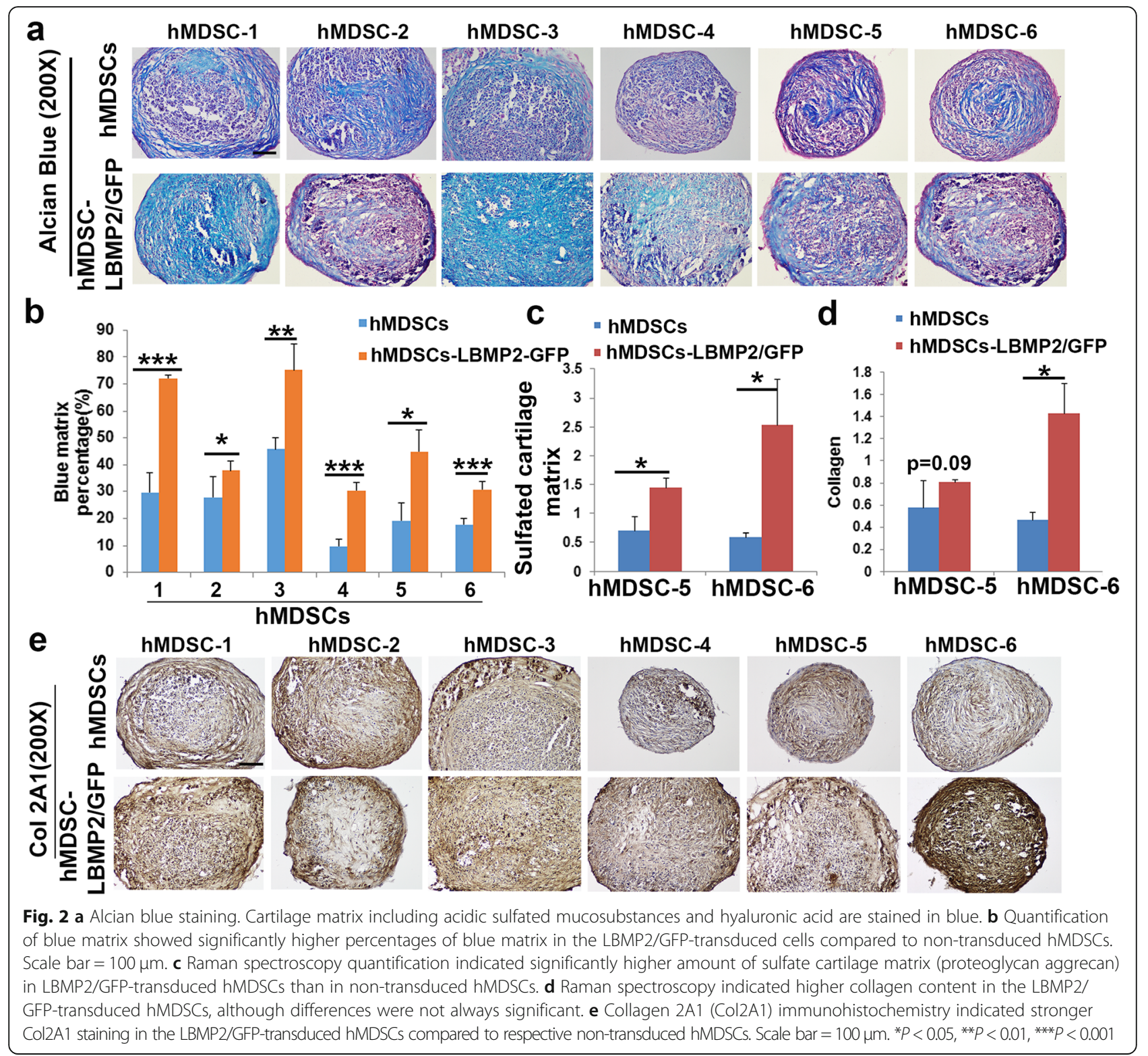

transduced hMDSCs contained the higher amount of sulfated cartilage matrix (proteoglycan aggrecan) and collagen when compared to non-transduced hMDSCs, although the differences were not always statistically significant (Fig. 2c, d). Col2A1 immunohistochemistry showed more Col2A1 in the LBMP2/GFP-transduced hMDSCs compared to non-transduced hMDSCs (Fig. 2e).

\section{MicroCT analyses revealed that cartilage erosion after} MIA-induced injury was decreased after treatment with hMDSCs with BMP2

MicroCT 3D images showed that each of the non-injured knee joints had smooth joint surfaces. The trochlear groove was smooth. The injured knees had different extents of joint cartilage erosion, as the subchondral bones were obviously visible in the trochlear groove and femoral condyles and tibia plateau. Knee joint space changed in the injured side of the knees in all groups compared to the non-injured side of knee (Fig. 3a-e). Quantification of knee joint space differences (injured knee - non-injured knee) indicated that the hMDSCLBMP2/GFP group and the BMP2 + sFLT1 + coacervate + hMDSC-LGFP group had significantly smaller joint space difference which is indicative of better healing compared to the PBS + coacervate group (Fig. 3f). No significant differences were found for the other groups.

Histology grade scores were lower in all the hMDSCinjected groups

We performed Toluidine blue and Alcian blue staining and histology scoring using the OARSI grading system 


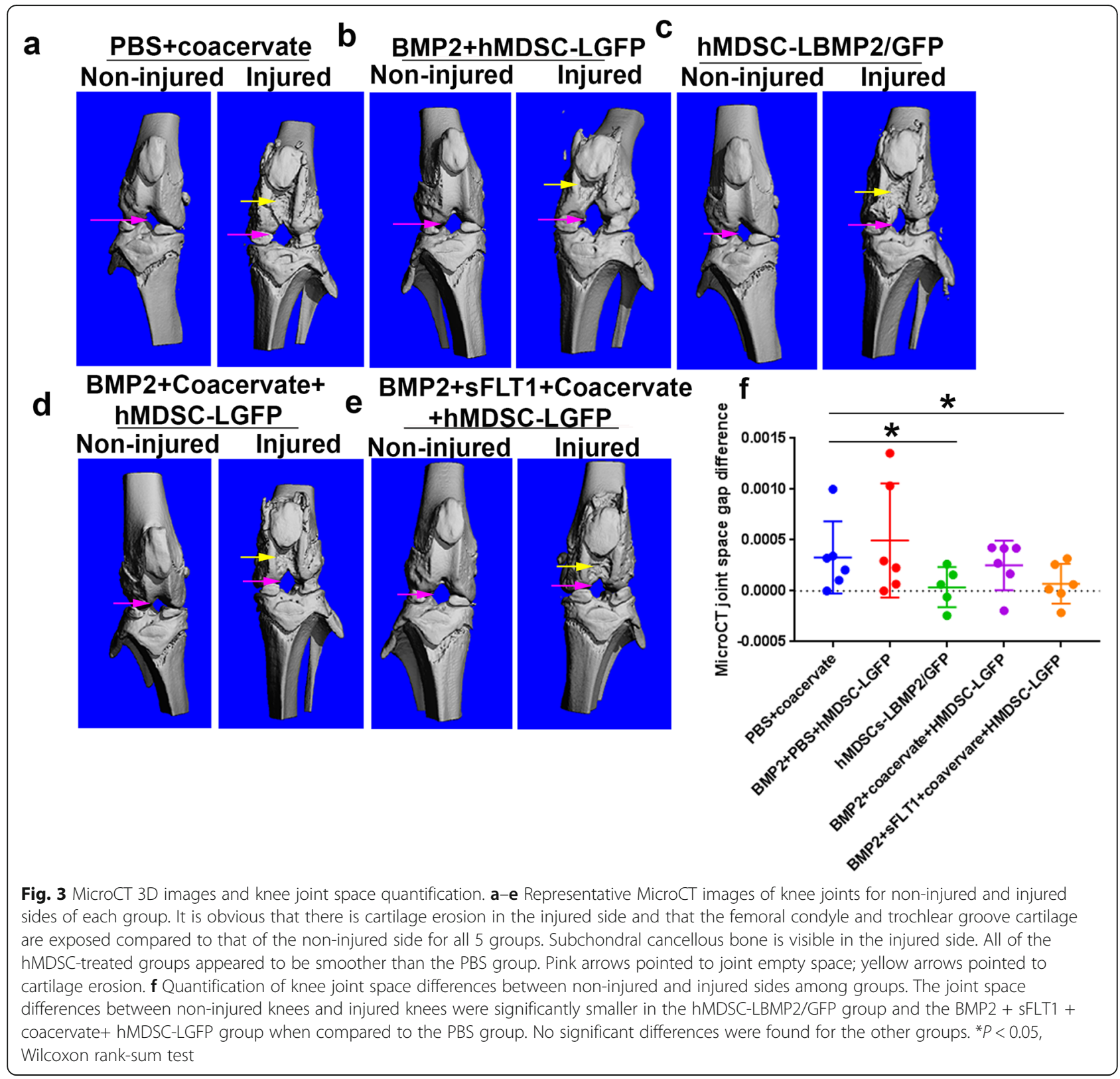

[34] to measure the histology grade of the knee joint cartilage, including the distal femur and proximal tibia articular cartilage. This system included 6 histological grades and 4 histological stages. The total score (grade multiplied by stage) ranged from 1 point (normal articular cartilage) to 24 points (no repair). The results indicated that all hMDSC-injected groups showed significant cartilage repair relative to the PBS + coacervate group that had no cells injected. There were minimal chondrocytes on the cartilage surface of the PBS + coacervate group from both edges of articular cartilage. In the PBS + BMP2 + hMDSC-LGFP group, we found chondrocytes clustered on the cartilage surface from both edges of the cartilage surface. In the hMDSC-
LBMP2/GFP group, we found more Toluidine bluepositive chondrocytes at the cartilage layer. In the BMP2 + coacervate + hMDSC-LGFP group, we observed more regenerated chondrocytes on the cartilage surface. Finally, in the BMP2 + sFLT1 + coacervate + hMDSCLGFP group, we observed more newly generated chondrocytes at the cartilage surface than in the PBS + coacervate group (Fig. 4a). However, none of the groups showed completely cartilage regeneration on the cartilage surface. Quantification of the histology scores showed that all the hMDSC-treated groups demonstrated significantly improved histology scores (lower value) compared to the $\mathrm{PBS}+$ coacervate group. Coacervate-delivered BMP2 plus hMDSCs achieved 

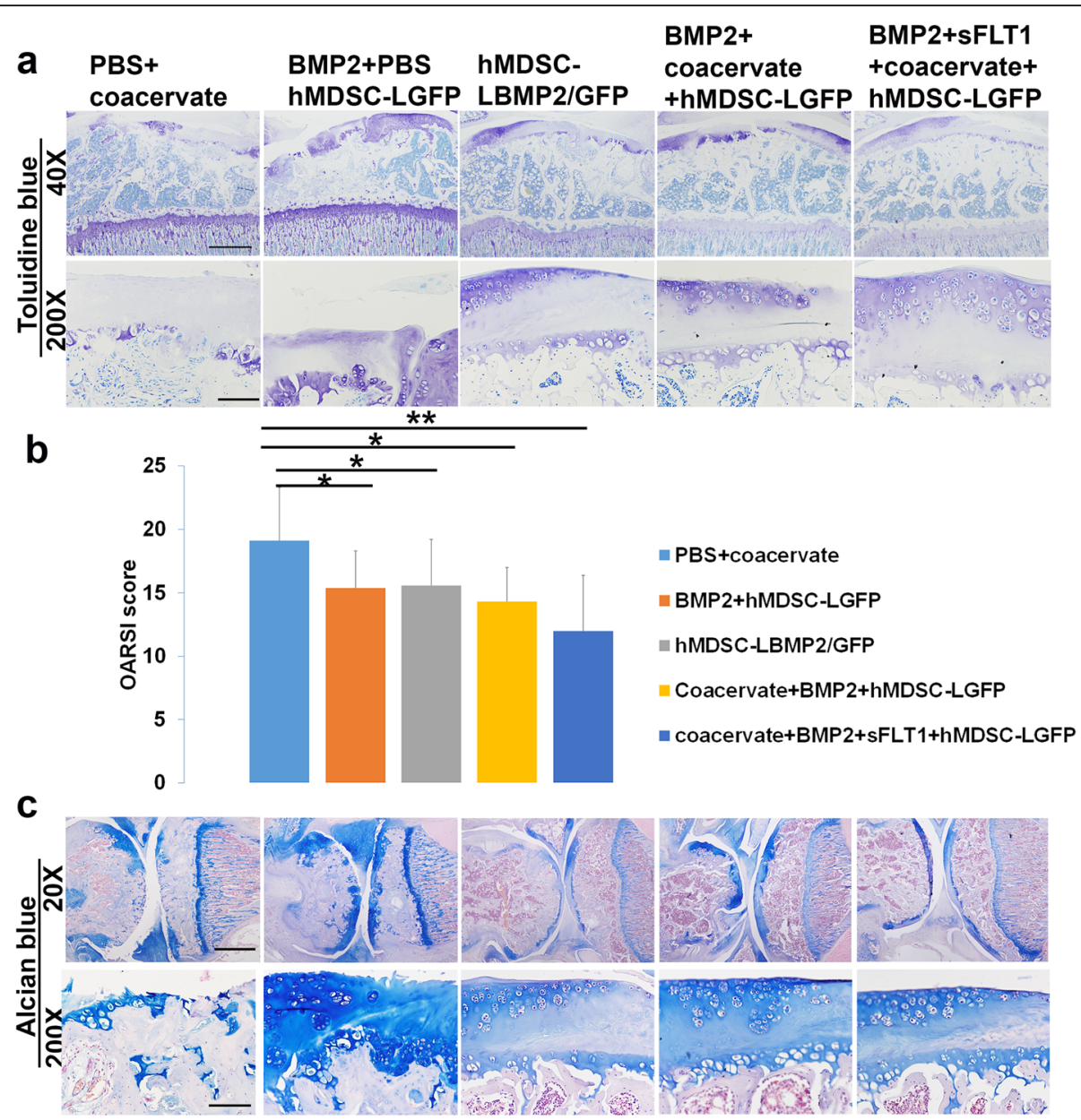

Fig. 4 Evaluation of histology score using Toluidine blue and Alcian blue staining. a Toluidine blue staining of the 5 different groups at 3 months after cell (or PBS + coacervate) injection. Upper panel showed entire tibia plateau at the condyle axial level, scale bar $=500 \mu \mathrm{m}$. Lower panel showed high magnification, scale bar $=100 \mu \mathrm{m}$. The PBS + coacervate group showed that the knee joint cartilage layer was almost completely destroyed. Very limited chondrocytes were left at the joint cartilage. All other groups showed cartilage regeneration at cartilage surface to different extent. b OARSI histology scores based on Toluidine blue staining in each of the 5 groups. All groups that were injected with hMDSCs had lower scores compared to the PBS + coacervate control group. ${ }^{*} P<0.05,{ }^{*} P<0.01$. Wilcoxon rank-sum test. $\mathbf{c}$ Alcian blue staining showed similar results as Toluidine blue staining. Upper panel showed the morphology of cartilage of entire knee at condyle axial level. Scale bar $=1000 \mu \mathrm{m}$. Lower panel showed chondrocytes morphology at the articular surface of each group, scale bar $=100 \mu \mathrm{m}$

slightly better scores than the hMDSC-LBMP2/GFP group but showed no statistical differences. The BMP2 + sFLT1 + coacervate + hMDSC-LGFP group yielded the best histology scores (Fig. 4b). Alcian blue staining demonstrated similar results as Toluidine blue staining (Fig. 4c).

Cartilage repair was improved in hMDSC-LBMP2/GFP and coacervate-delivered BMP2 and sFlt1 in combination with hMDSCs groups

We performed H\&E staining to look at the general articular cartilage structure for each group. We found that the PBS + coacervate control group had very few chondrocytes in the cartilage area from both edges of articular cartilage, which implied that the majority of the cartilage cells had died. Subchondral bone was exposed in some areas. In the BMP2 + hMDSC-LGFP group, we found regenerated chondrocytes in clusters. Regenerated AC with chondrocytes was observed, but there was still much of the joint surface without cells. In the hMDSC-LBMP2/GFP group, we found more $\mathrm{AC}$ areas with regenerated cartilage cells. In the BMP2 + coacervate + hMDSC-LGFP group, we found similar articular cartilage regeneration compared to the hMDSC-LBMP2/GFP group. There were more cells in the superficial zone and fewer cells in the middle zone. In the BMP2 + sFLT1 + coacervate + hMDSC-LGFP group, we found better articular cartilage structure (Fig. 5a). Of note, we did not find residual coacervate in the knee cartilage after 12 weeks 


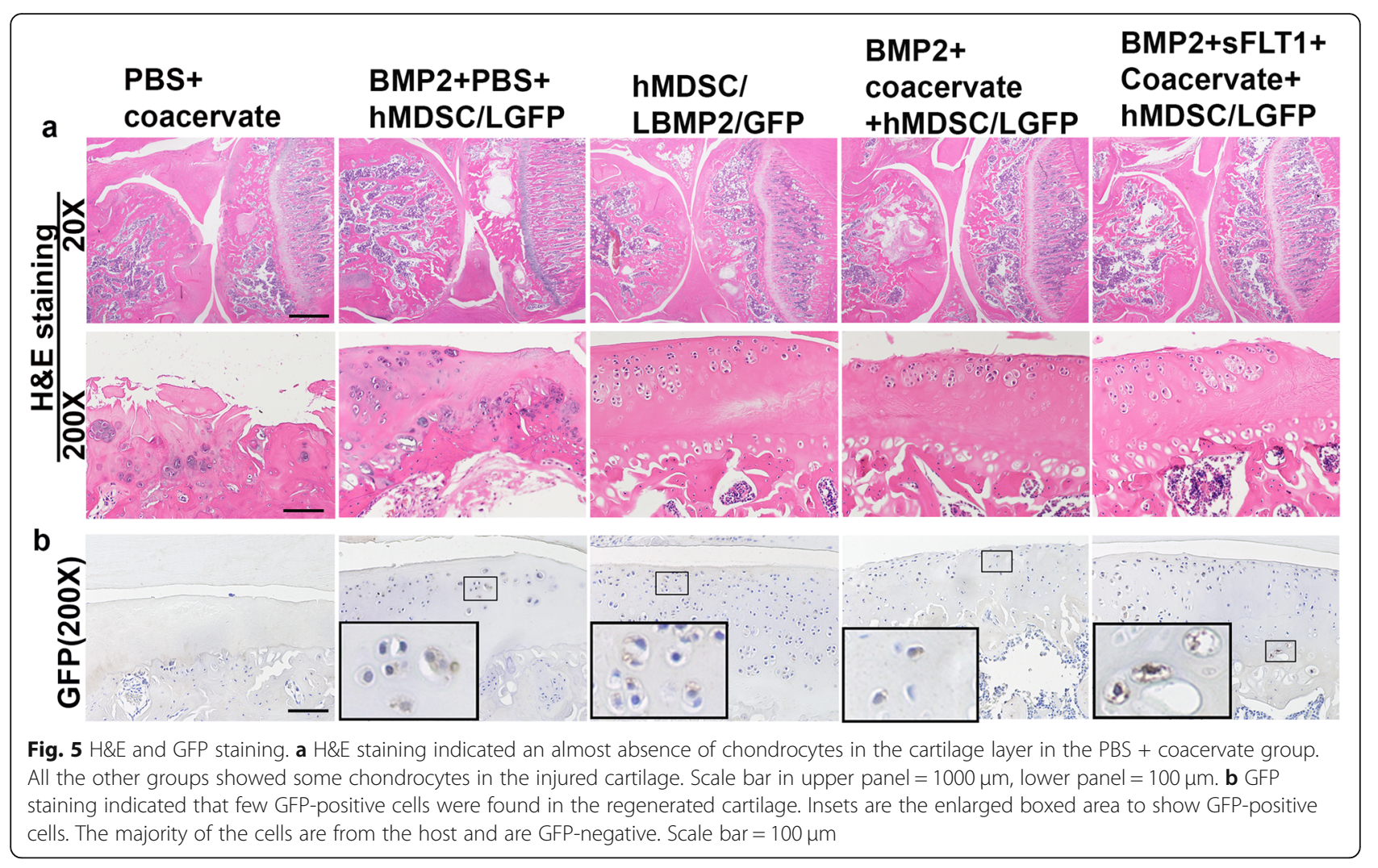

post-injection, which indicated that the coacervate was absorbed.

Donor cells contributed to the regenerated cartilage, but host cells contributed the majority of regenerated chondrocytes

In order to trace whether the transplanted hMDSCs differentiated into chondrocytes and contributed to the regenerated cartilage, we performed GFP immunohistochemistry. We found scattered GFP-positive cells in the regenerated cartilage area on the articular surface in all the groups that had hMDSCs injected. However, GFP-positive cells were relatively fewer in number compared to GFP-negative cells indicating that the regenerated cartilage is composed primarily of host-derived chondrocytes (Fig. 5b). Insets are the enlarged boxed area from the original image showing the GFP+ cells.

\section{Col2 staining demonstrated the regeneration of chondrocytes in the regenerated cartilage surface} In order to test whether the newly generated cartilage on AC surface has cartilage properties, we performed Col2 staining, which labels typical cartilage matrix. We observed few chondrocytes at the edge of articular cartilage in the PBS + coacervate group that are Col2-positive, and the residual matrix was less intense Col 2-positive. In the BMP2 + hMDSC-LGFP group, we found chondrocyte regeneration, although only partial, and Col2 staining was strongly positive in certain areas. In the hMDSC-LBMP2/ GFP group, we found more chondrocytes that were Col2positive on the articular cartilage surface. In the BMP2 + coacervate + hMDSC-LGFP group, we also observed extensive expression of $\mathrm{Col} 2$ in the regenerated cartilage. Many Col2-positive chondrocytes were identified. In the BMP2 + sFLT1 + hMDSC-LGFP group, there were more Col2-positive chondrocytes compared to other groups (Fig. 6).

\section{Discussion}

In this study, we investigated chondrogenic potential of 6 human populations of hMDSCs and found that the LBMP2-transduced hMDSCs exhibited enhanced chondrogenic differentiation in vitro compared to the nontransduced cells. Furthermore, we tested the cartilage repair capacity of hMDSC-LGFP plus free BMP2 and hMDSC-LGFP combined with coacervate-delivered BMP2, and compared the results with hMDSC-LBMP2/ GFP group to test their cartilage repair capacity in vivo. We also tested dual delivery of BMP2 and sFLT1 with the coacervate for cartilage repair. Our results indicated that all the hMDSC injection groups demonstrated partial cartilage repair, although to different extents. The hMDSCLBMP2/GFP group showed both improvement in cartilage erosion (revealed by microCT 3D images of joint gaps 


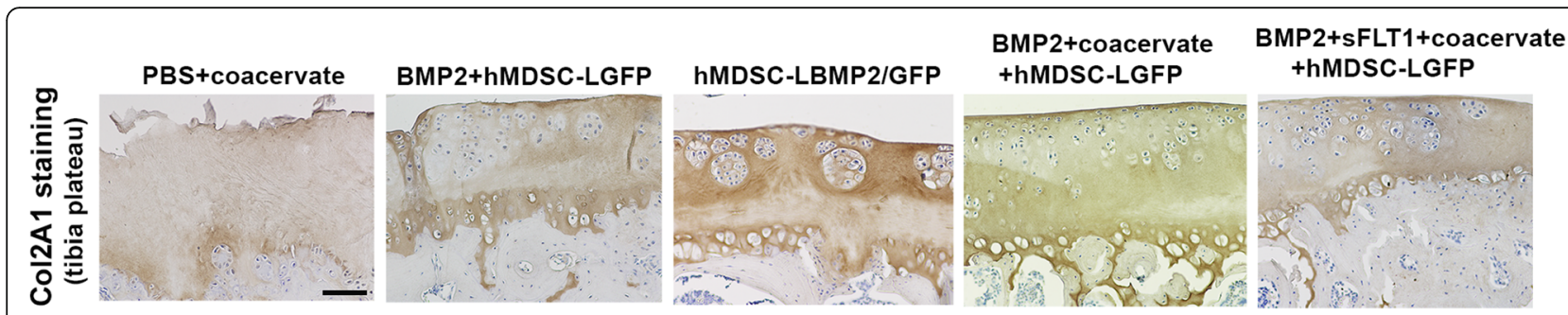

Fig. 6 Col2 staining. Brown color showed Col2-positive cartilage matrix. We observed very few chondrocytes in the PBS + coacervate group, and the residual matrix was Col2A1-positive. The BMP2 + hMDSC-LGFP group showed chondrocyte regeneration, although only partial, and Col2A1 staining was positive in certain areas. In the hMDSC-LBMP2/GFP group, Col2-positive chondrocytes were found in cluster. In the BMP2 + coacervate + hMDSCLGFP group, Col2-positive chondrocytes distributed mainly on the superfacial zone and middle zone, zones near tide marker showed no chondrocytes. The BMP2 + sFLT1 + hMDSC-LGFP group showed slightly better chondrocytes distribution than the hMDSC-LBMP2/GFP group and BMP2 + coacervate+ hMDSCLGFP group. Scale bar $=100 \mu \mathrm{m}$

and histological score) relative to the PBS + coacervate group. Coacervate-delivered BMP2 plus hMDSC-LGFP achieved similar histology scores compared to the hMDSC-LBMP2/GFP gene therapy approach. Coacervate dual delivery of BMP2 and sFLT1 plus hMDSC-LGFP achieved the best $\mathrm{AC}$ regeneration observed by microCT and histology scores. Many different stem cells have been used in the treatment of arthritis experimentally or clinical trial. Bone marrow aspirate concentrate embedded in hyaluronic acid scaffold has been used for cartilage injury in clinic and proved to be good to excellent outcome at long-term follow-up for small to large cartilage lesions, and single to multiple lesions [35]. Mesenchymal stem cells loaded on Tantalum have been shown to improve cartilage repair in a large osteochondral defect model in goat [36]. Adipose stem cells in combination with different scaffold have been shown to ameliorate cartilage regeneration in a different animal models [37-39]. Adipose stem cells have been tested in phase II clinical trial for knee osteoarthritis treatment, and the results have indicated the improvement of joint function, pain, quality of life, and cartilage regeneration [40]. Adipose stem cells have also been shown beneficial effect for microfracture-mediated cartilage repair in clinical patient [41]. Previously, we have shown that murine MDSCs can efficiently repair rat MIAinduced osteoarthritis $[12,42]$ when transduced with retroviral-BMP4/GFP. Our current study presents findings for the first time the use of human MDSCs to repair cartilage. In this study, we first tested whether hMDSC-BMP2/ GFP has enhanced chondrogenic differentiation ability. We tested 6 populations of hMDSCs and found that LBMP2/GFP-transduced cells all demonstrated enhanced in vitro chondrogenesis compared to non-transduced cells, as indicated by Alcian blue and Col2A staining as well as Raman spectrometry quantification of sulfated cartilage matrix and collagen. These results encouraged us to perform in vivo experiments. Furthermore, we found that all groups, which had hMDSC treatments, showed improved AC repair compared to the PBS control group. We found that the hMDSC-LBMP2/GFP group had better cartilage repair than the PBS + coacervate group, which demonstrated limited cartilage repair after MIA-induced osteoarthritis. However, the direct contribution of the hMDSCs to the repaired cartilage was limited, as only a few GFP-positive cells were found in the repaired cartilage. Several reasons may explain the lower GFP detection rate on the repaired cartilage. One possibility is that the GFP from the transduced hMDSCs is being gradually lost during cell division. It is also possible because the GFPpositive cell percentage is not $100 \%$ even after cell sorting. The loss of GFP expression may also be attributed to the long period of decalcification, since for this study we have decalcified for 3 months using a 10\% EDTA plus 1\% $\mathrm{NaOH}$ solution. We only decalcified for 1 month for regenerated bone tissues mediated by murine MDSC/ BMP4/GFP $[15,30]$. However, we have ruled out the possibility of the GFP antibody as we have used this antibody for murine MDSCs /BMP4GFP transplantation and have shown robust GFP-positive staining $[15,30]$. The hMDSCs mainly served as a BMP2 delivery vehicle. Donor cell contribution to cartilage repair has been shown to vary by the use of different stem cells. It has been shown that murine MDSCs transduced with BMP4/GFP can directly differentiate into chondrocytes in MIA-induced osteoarthritis [12] and osteochondral defects [43], but also in a small percentage compared to those from host cells. Bone marrow mesenchymal stem cells injected in the knee joint in MIA-induced model reduced pain, but did not improve structural damage to the cartilage and subchondral bone and synovitis. Cell tracking indicated cell survival until 2 weeks [44]. While there has been concern with respect to gene therapy and biosafety, we, therefore, also tested whether using biomaterials to deliver BMP2 can also achieve similar cartilage repair effects of lenti-BMP2transduced hMDSCs. We used coacervate, a polymer biomaterial that has been shown to successfully deliver other growth factors for tissue repair [32]. Indeed, we found that using coacervate to deliver $500 \mathrm{ng}$ BMP2 in combination 
with hMDSC-LGFP (BMP2 + coacervate + hMDSC-LGFP group) achieved similar cartilage repair in terms of microCT analysis and histology scores as lenti-BMP2/ GFP-transduced hMDSCs. The biomaterial is injectable and with no residue found in the injected joint 12 weeks after intra-articular injection. Furthermore, we took advantage of the biomaterial's ability to deliver growth factors and performed dual delivery of BMP2 (500 ng) and sFLT1 (500 ng), as it is known that blocking angiogenesis can further enhance cartilage repair [12, 13]. Our results demonstrated that adding sFLT1 enhanced cartilage repair with hMDSCs and BMP2, as demonstrated by microCT and histology. These results support the feasibility of using hMDSCs and growth factor without the need of gene transfer for cartilage repair. Finally, we need to point out that none of the treatment regimen was able to completely repair entire damaged cartilage surface. The possible reason may be because the disease progression continues until 12 weeks after MIA-induced arthritis [45]. The other reason may be due to the fact when the cells were injected into the joint space, and only those cells that attached to the cartilage surface will participate in the cartilage repair. Other injected cells may only exert paracrine effect. Therefore, more researches are warranted to further enhance the cartilage repair mediated by hMDSCs in combination with growth factors such as optimization of dose of BMPs and cell numbers and improvement of cell survival and the use multiple injections.

\section{Conclusion}

We found LBMP2/GFP-transduced hMDSCs have enhanced chondrogenic capacity compared to nontransduced hMDSCs in vitro. The delivery of BMP2 together with hMDSCs significantly improved cartilage repair. The delivery of BMP2 using both gene therapy and coacervate biomaterial enhanced hMDSC-mediated cartilage repair. Blocking angiogenesis by co-delivery of sFLT1 along with BMP2 plus hMDSC-LGFP achieved the best results for cartilage repair. Endogenous cells recruited by BMP2 contributed more to the cartilage repair than the transplanted cells. Our results support the application of hMDSCs for cartilage repair using both gene therapy and biomaterials to deliver growth factors.

\section{Abbreviations \\ MDSC: Muscle-derived stem cells; BMP2: Bone morphogenetic protein 2; GFP: Green fluorescent protein; MicroCT: Micro-computed tomography; Col2: Collagen 2; sFLT1: Soluble fms-like tyrosine kinase-1; \\ PEAD: Polyethylene argininylaspartate diglyceride; MIA: Monoiodoacetate; IRES: Internal ribosomal entry site; FACS: Fluorescent activated cell sorting; PBS: Phosphate-buffered saline; ANOVA: Analysis of variance; \\ DAB: Diaminobenzidine; NBF: Neutral buffered formalin}

\section{Acknowledgements}

We thank Dr. Mary Hall and Dr. William Rodkey for proofreading the paper. We also thank Mrs. Anna-Laura Nelson, M.S., for proof reading during manuscript revision.

\section{Authors' contributions}

XG was responsible for the experimental design, performed the majority of experiments in vitro and in vivo, drafted the manuscript, and gave final approval of the manuscript. HC participated in the animal model creation, animal monitoring and sacrifice, and tissue harvesting. HA synthesized the coacervate biomaterials and was responsible for the quality control. YT constructed lenti-GFP and lenti-BMP2/GFP vectors and preparation of virus. SA performed the tissue paraffin processing, embedding, and some sectioning. AL isolated hMDSCs. XS assisted with animal sacrifice and some microCT scanning. GL performed the Raman spectroscopy analysis for pellets. $\mathrm{CH}$ assisted with staining. BW designed the lenti-GFP and lenti-BMP2/GFP vectors. XB provided Raman spectroscopy experiment design. YW was responsible for the concept, provided coacervate materials, was responsible for the revision of the manuscript, and gave final approval of the manuscript. JH was responsible for the concept, experimental design, and revision of the manuscript and gave final approval of the manuscript. All authors read and approved the final manuscript.

\section{Funding}

This project was supported by a R21 grant awarded to Dr. Johnny Huard (R21AR066206).

\section{Availability of data and materials}

The datasets used or analyzed (or both) during the current study are available from the corresponding author on reasonable request.

\section{Ethics approval and consent to participate}

The use of human tissues was approved by the Institutional Review Board (IRB) of the University of Texas Health Science Center at Houston (UTHealth), and all animal experiments and procedures were approved by the Institutional Animal Care and Use Committee (IACUC) of the UTHealth (Protocol number: AWC-15-0072). UTHealth was accredited by Association for Assessment and ACcreditation of Laboratory Animal Care (AAALAC). The use of animals for in vivo study followed the guidelines of "Basel Declaration" and "ethical guidelines" of the International Council for Laboratory Animal Science (ICLAS).

Consent for publication

Not applicable.

\section{Competing interests}

The authors declare that they have no competing interests.

\section{Author details}

'Department of Orthopaedic Surgery, University of Texas Health Science Center at Houston, Houston, TX, USA. Institute of Molecular Medicine, McGovern Medical School, University of Texas Health Science Center at Houston, Houston, TX, USA. ${ }^{3}$ Department of Center for Regenerative Sports Medicine, Steadman Philippon Research Institute, Vail, CO, USA. ${ }^{4}$ Meinig School of Biomedical Engineering, Cornell University, Ithaca, NY, USA. ${ }^{5}$ Department of Orthopaedic Surgery, University of Pittsburgh, Pittsburgh, PA, USA. ${ }^{6}$ Department of Nanomedicine, Institute of Molecular Medicine, McGovern Medical School, University of Texas Health Science Center at Houston, Houston, TX, USA.

Received: 22 May 2019 Revised: 31 July 2019

Accepted: 30 September 2019 Published online: 26 November 2019

References

1. Xie X, Wang Y, Zhao C, Guo S, Liu S, Jia W, et al. Comparative evaluation of MSCs from bone marrow and adipose tissue seeded in PRP-derived scaffold for cartilage regeneration. Biomaterials. 2012;33(29):7008-18. https://doi.org/ 10.1016/j.biomaterials.2012.06.058

2. Sieker JT, Kunz M, Weissenberger M, Gilbert F, Frey S, Rudert M, et al. Direct bone morphogenetic protein 2 and Indian hedgehog gene transfer for articular cartilage repair using bone marrow coagulates. Osteoarthr Cartil. 2015;23(3):433-42. https://doi.org/10.1016/j.joca.2014.11.008.

3. Liu H, Ding J, Wang C, Wang J, Wang Y, Yang M, et al. Intra-articular transplantation of allogeneic BMMSCs rehabilitates cartilage injury of antigen-induced arthritis. Tissue Eng Part A. 2015;21(21-22):2733-43. https:// doi.org/10.1089/ten.TEA.2014.0666. 
4. Goodrich LR, Chen AC, Werpy NM, Williams AA, Kisiday JD, Su AW, et al. Addition of mesenchymal stem cells to autologous platelet-enhanced fibrin scaffolds in chondral defects: does it enhance repair? J Bone Joint Surg Am. 2016;98(1):23-34. https://doi.org/10.2106/JBJS.0.00407.

5. Dragoo JL, Carlson G, McCormick F, Khan-Farooqi H, Zhu M, Zuk PA, et al. Healing full-thickness cartilage defects using adipose-derived stem cells. Tissue Eng. 2007;13(7):1615-21. https://doi.org/10.1089/ten.2006.0249.

6. Zhang K, Zhang Y, Yan S, Gong L, Wang J, Chen X, et al. Repair of an articular cartilage defect using adipose-derived stem cells loaded on a polyelectrolyte complex scaffold based on poly(l-glutamic acid) and chitosan. Acta Biomater. 2013;9(7):7276-88. https://doi.org/10.1016/j.actbio. 2013.03.025.

7. $\quad L u C H$, Yeh TS, Yeh CL, Fang YH, Sung LY, Lin SY, et al. Regenerating cartilages by engineered ASCs: prolonged TGF-beta3/BMP-6 expression improved articular cartilage formation and restored zonal structure. Mol Ther. 2014;22(1):186-95. https://doi.org/10.1038/mt.2013.165.

8. Lee JM, Im GI. SOX trio-co-transduced adipose stem cells in fibrin gel to enhance cartilage repair and delay the progression of osteoarthritis in the rat. Biomaterials. 2012;33(7):2016-24. https://doi.org/10.1016/j.biomaterials. 2011.11.050

9. Cui L, Wu Y, Cen L, Zhou H, Yin S, Liu G, et al. Repair of articular cartilage defect in non-weight bearing areas using adipose derived stem cells loaded polyglycolic acid mesh. Biomaterials. 2009;30(14):2683-93. https://doi.org/10. 1016/j.biomaterials.2009.01.045.

10. Goldberg A, Mitchell K, Soans J, Kim L, Zaidi R. The use of mesenchymal stem cells for cartilage repair and regeneration: a systematic review. J Orthop Surg Res. 2017;12(1):39. https://doi.org/10.1186/s13018-017-0534-y.

11. Kuroda R, Usas A, Kubo S, Corsi K, Peng H, Rose T, et al. Cartilage repair using bone morphogenetic protein 4 and muscle-derived stem cells. Arthritis Rheum. 2006;54(2):433-42. https://doi.org/10.1002/art.21632.

12. Matsumoto T, Cooper GM, Gharaibeh B, Meszaros LB, Li G, Usas A, et al. Cartilage repair in a rat model of osteoarthritis through intraarticular transplantation of muscle-derived stem cells expressing bone morphogenetic protein 4 and soluble Flt-1. Arthritis Rheum. 2009;60(5): 1390-405. https://doi.org/10.1002/art.24443.

13. Kubo S, Cooper GM, Matsumoto T, Phillippi JA, Corsi KA, Usas A, et al. Blocking vascular endothelial growth factor with soluble Flt-1 improves the chondrogenic potential of mouse skeletal muscle-derived stem cells. Arthritis Rheum. 2009;60(1):155-65. https://doi.org/10.1002/art.24153.

14. Gao X, Usas A, Lu A, Tang Y, Wang B, Chen CW, et al. BMP2 is superior to BMP4 for promoting human muscle-derived stem cell-mediated bone regeneration in a critical-sized calvarial defect model. Cell Transplant. 2013: 22(12):2393-408. https://doi.org/10.3727/096368912X658854.

15. Gao X, Usas A, Proto JD, Lu A, Cummins JH, Proctor A, et al. Role of donor and host cells in muscle-derived stem cell-mediated bone repair: differentiation vs. paracrine effects. FASEB J. 2014;28(8):3792-809. https://doi. org/10.1096/fj.13-247965.

16. Gao X, Lu A, Tang Y, Schneppendahl J, Liebowitz AB, Scibetta AC, et al. Influences of donor and host age on human muscle-derived stem cellmediated bone regeneration. Stem Cell Res Ther. 2018;9(1):316. https://doi. org/10.1186/s13287-018-1066-Z.

17. Berne AS, Dato C, Mason DJ, Rafferty M. A nursing model for addressing the health needs of homeless families. Image J Nurs Sch. 1990;22(1):8-13.

18. Chu H, Chen CW, Huard J, Wang Y. The effect of a heparin-based coacervate of fibroblast growth factor-2 on scarring in the infarcted myocardium. Biomaterials. 2013;34(6):1747-56. https://doi.org/10.1016/j. biomaterials.2012.11.019.

19. Johnson NR, Wang Y. Controlled delivery of heparin-binding EGF-like growth factor yields fast and comprehensive wound healing. I Control Release. 2013;166(2):124-9. https://doi.org/10.1016/j.jconrel.2012.11.004.

20. Johnson NR, Wang Y. Controlled delivery of sonic hedgehog morphogen and its potential for cardiac repair. PLoS One. 2013;8(5):e63075. https://doi. org/10.1371/journal.pone.0063075.

21. Awada HK, Johnson NR, Wang Y. Sequential delivery of angiogenic growth factors improves revascularization and heart function after myocardial infarction. J Control Release. 2015;207:7-17. https://doi.org/10.1016/j.jconrel. 2015.03.034.

22. Johnson NR, Wang Y. Coacervate delivery of HB-EGF accelerates healing of type 2 diabetic wounds. Wound Repair Regen. 2015;23(4):591-600. https:// doi.org/10.1111/wrr.12319.
23. Lee MS, Ahmad T, Lee J, Awada HK, Wang Y, Kim K, et al. Dual delivery of growth factors with coacervate-coated poly(lactic-co-glycolic acid) nanofiber improves neovascularization in a mouse skin flap model. Biomaterials. 2017;124:65-77. https://doi.org/10.1016/j.biomaterials.2017. 01.036 .

24. Long DW, Johnson NR, Jeffries EM, Hara H, Wang Y. Controlled delivery of platelet-derived proteins enhances porcine wound healing. J Control Release. 2017;253:73-81. https://doi.org/10.1016/j.jconrel. 2017.03.021.

25. Li H, Johnson NR, Usas A, Lu A, Poddar M, Wang Y, et al. Sustained release of bone morphogenetic protein 2 via coacervate improves the osteogenic potential of muscle-derived stem cells. Stem Cells Transl Med. 2013;2(9): 667-77. https://doi.org/10.5966/sctm.2013-0027.

26. Lavasani M, Lu A, Thompson SD, Robbins PD, Huard J, Niedernhofer LJ. Isolation of muscle-derived stem/progenitor cells based on adhesion characteristics to collagen-coated surfaces. Methods Mol Biol. 2013;976:5365. https://doi.org/10.1007/978-1-62703-317-6_5.

27. Yaffe D, Saxel O. Serial passaging and differentiation of myogenic cells isolated from dystrophic mouse muscle. Nature. 1977;270(5639):725-7. https://doi.org/10.1038/270725a0.

28. Yaffe D. Retention of differentiation potentialities during prolonged cultivation of myogenic cells. Proc Natl Acad Sci U S A. 1968;61(2):477-83. https://doi.org/10.1073/pnas.61.2.477.

29. Rando TA, Blau HM. Primary mouse myoblast purification, characterization, and transplantation for cell-mediated gene therapy. J Cell Biol. 1994;125(6): $1275-87$.

30. Gao X, Usas A, Lu A, Kozemchak A, Tang Y, Poddar M, et al. Cyclooxygenase-2 deficiency impairs muscle-derived stem cell-mediated bone regeneration via cellular autonomous and non-autonomous mechanisms. Hum Mol Genet. 2016;25(15):3216-31. https://doi.org/10.1093/ hmg/ddw172.

31. Chu H, Johnson NR, Mason NS, Wang Y. A [polycation:heparin] complex releases growth factors with enhanced bioactivity. J Control Release. 2011; 150(2):157-63. https://doi.org/10.1016/j.jconrel.2010.11.025.

32. Chu H, Gao J, Chen CW, Huard J, Wang Y. Injectable fibroblast growth factor-2 coacervate for persistent angiogenesis. Proc Natl Acad Sci U S A. 2011;108(33):13444-9. https://doi.org/10.1073/pnas.1110121108.

33. Chen WC, Lee BG, Park DW, Kim K, Chu H, Kim K, et al. Controlled dual delivery of fibroblast growth factor-2 and Interleukin-10 by heparin-based coacervate synergistically enhances ischemic heart repair. Biomaterials. 2015. 72:138-51. https://doi.org/10.1016/j.biomaterials.2015.08.050.

34. Pritzker KP, Gay S, Jimenez SA, Ostergaard K, Pelletier JP, Revell PA, et al. Osteoarthritis cartilage histopathology: grading and staging. Osteoarthr Cartil. 2006;14(1):13-29. https://doi.org/10.1016/j.joca.2005.07.014.

35. Gobbi A, Whyte GP. Long-term clinical outcomes of one-stage cartilage repair in the knee with hyaluronic acid-based scaffold embedded with mesenchymal stem cells sourced from bone marrow aspirate concentrate. Am J Sports Med 2019:363546519845362. doi:https://doi.org/10.1177/ 0363546519845362

36. Wei X, Liu B, Liu G, Yang F, Cao F, Dou X, et al. Mesenchymal stem cellloaded porous tantalum integrated with biomimetic 3D collagen-based scaffold to repair large osteochondral defects in goats. Stem Cell Res Ther. 2019:10(1):72. https://doi.org/10.1186/s13287-019-1176-2.

37. Beigi MH, Atefi A, Ghanaei HR, Labbaf S, Ejeian F, Nasr-Esfahani MH. Activated platelet-rich plasma improves cartilage regeneration using adipose stem cells encapsulated in a 3D alginate scaffold. J Tissue Eng Regen Med. 2018;12(6):1327-38. https://doi.org/10.1002/term.2663.

38. Huang S, Song X, Li T, Xiao J, Chen Y, Gong X, et al. Pellet coculture of osteoarthritic chondrocytes and infrapatellar fat pad-derived mesenchymal stem cells with chitosan/hyaluronic acid nanoparticles promotes chondrogenic differentiation. Stem Cell Res Ther. 2017;8(1):264. https://doi. org/10.1186/s13287-017-0719-7.

39. Spakova T, Amrichova J, Plsikova J, Harvanova D, Hornak S, Ledecky V, et al. A preliminary study comparing microfracture and local adherent transplantation of autologous adipose-derived stem cells followed by Intraarticular injection of platelet-rich plasma for the treatment of chondral defects in rabbits. Cartilage. 2018;9(4):410-6. https://doi.org/10.1177/ 1947603517713816.

40. Lu L, Dai C, Zhang Z, Du H, Li S, Ye P, et al. Treatment of knee osteoarthritis with intra-articular injection of autologous adipose-derived mesenchymal progenitor cells: a prospective, randomized, double-blind, active-controlled, 
phase Ilb clinical trial. Stem Cell Res Ther. 2019;10(1):143. https://doi.org/10. 1186/s13287-019-1248-3.

41. Koh YG, Kwon OR, Kim YS, Choi YJ, Tak DH. Adipose-derived mesenchymal stem cells with microfracture versus microfracture alone: 2-year follow-up of a prospective randomized trial. Arthroscopy. 2016;32(1):97-109. https://doi. org/10.1016/j.arthro.2015.09.010

42. Matsumoto T, Kubo S, Meszaros LB, Corsi KA, Cooper GM, Li G, et al. The influence of sex on the chondrogenic potential of muscle-derived stem cells: implications for cartilage regeneration and repair. Arthritis Rheum. 2008;58(12):3809-19. https://doi.org/10.1002/art.24125.

43. Li H, Lu A, Tang Y, Beckman S, Nakayama N, Poddar M, et al. The superior regenerative potential of muscle-derived stem cells for articular cartilage repair is attributed to high cell survival and chondrogenic potential. Mol Ther Methods Clin Dev. 2016;3:16065. https://doi.org/10.1038/mtm.2016.65.

44. van Buul GM, Siebelt M, Leijs MJ, Bos PK, Waarsing JH, Kops N, et al. Mesenchymal stem cells reduce pain but not degenerative changes in a mono-iodoacetate rat model of osteoarthritis. J Orthop Res. 2014;32(9): 1167-74. https://doi.org/10.1002/jor.22650

45. Udo M, Muneta T, Tsuji K, Ozeki N, Nakagawa Y, Ohara T, et al. Monoiodoacetic acid induces arthritis and synovitis in rats in a dose- and time-dependent manner: proposed model-specific scoring systems. Osteoarthr Cartil. 2016;24(7): 1284-91. https://doi.org/10.1016/j.joca.2016.02.005.

\section{Publisher's Note}

Springer Nature remains neutral with regard to jurisdictional claims in published maps and institutional affiliations.

Ready to submit your research? Choose BMC and benefit from:

- fast, convenient online submission

- thorough peer review by experienced researchers in your field

- rapid publication on acceptance

- support for research data, including large and complex data types

- gold Open Access which fosters wider collaboration and increased citations

- maximum visibility for your research: over $100 \mathrm{M}$ website views per year

At $\mathrm{BMC}$, research is always in progress.

Learn more biomedcentral.com/submissions 